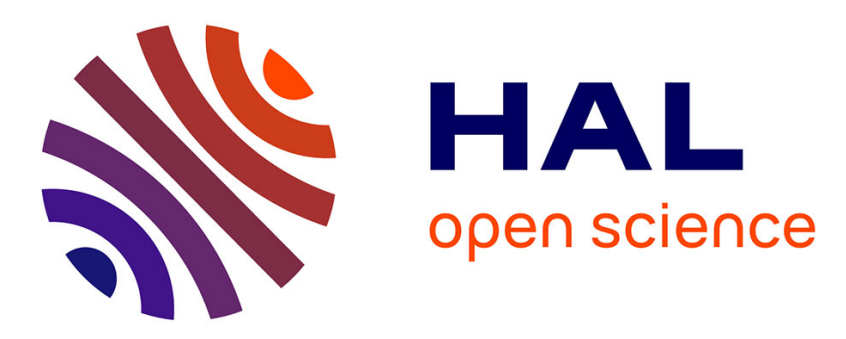

\title{
Caractéristiques morphologiques des constituants et comportement à la limite élastique d'un matériau biphasé Fe/Ag
}

T. Bretheau, Dominique Jeulin

\section{- To cite this version:}

T. Bretheau, Dominique Jeulin. Caractéristiques morphologiques des constituants et comportement à la limite élastique d'un matériau biphasé Fe/Ag. Revue de Physique Appliquée, 1989, 24 (9), pp.861-869. 10.1051/rphysap:01989002409086100 . jpa-00246109

\section{HAL Id: jpa-00246109 \\ https://hal.science/jpa-00246109}

Submitted on 1 Jan 1989

HAL is a multi-disciplinary open access archive for the deposit and dissemination of scientific research documents, whether they are published or not. The documents may come from teaching and research institutions in France or abroad, or from public or private research centers.
L'archive ouverte pluridisciplinaire HAL, est destinée au dépôt et à la diffusion de documents scientifiques de niveau recherche, publiés ou non, émanant des établissements d'enseignement et de recherche français ou étrangers, des laboratoires publics ou privés. 


\title{
Caractéristiques morphologiques des constituants et comportement à la limite élastique d'un matériau biphasé $\mathrm{Fe} / \mathrm{Ag}$
}

\author{
T. Bretheau $\left({ }^{1}\right)$ et D. Jeulin $\left({ }^{2}\right)$ \\ (1) LPMTM, Université Paris Nord, Avenue J. B. Clément, 93430 Villetaneuse, France \\ (2) Centre de Géostatistique, ENSMP, 35, rue Saint Honoré, 77305 Fontainebleau, France
}

(Reçu le 3 février 1989, révisé le 17 mai 1989, accepté le 8 juin 1989)

\begin{abstract}
Résumé. - Afin de mettre en évidence l'influence de la répartition spatiale des phases sur le comportement mécanique en plasticité, des matériaux biphasés fer/argent ont été élaborés par métallurgie des poudres. Des essais de traction réalisés dans toute la gamme de concentration ont permis de distinguer différents régimes de plastification, soit par propagation de bandes de Lüders pour les plus fortes concentrations en fer $(>70 \%)$ soit par plastification homogène pour les concentrations inférieures. Afin d'atteindre une meilleure caractérisation morphologique tridimensionnelle de ces matériaux, des mesures morphologiques bidimensionnelles ont été réalisées (sur un grand nombre de champs mais pour une gamme de concentration étroite) au moyen d'un dispositif d'analyse d'image. Ces mesures ont montré la légitimité d'une représentation de ces matériaux au moyen d'un schéma booléen dont les paramètres sont déterminés expérimentalement. Ceci donne accès à la variation du nombre de connexité tridimensionnelle en fonction de la concentration en fer dont la bonne corrélation avec les différents régimes de plastification montre bien l'influence déterminante de la répartition spatiale sur le comportement plastique global.
\end{abstract}

\begin{abstract}
In order to show off the influence of the phase space distribution on the mechanical behaviour in plasticity, iron/silver two phase materials have been elaborated via the powder technique. Tensile tests performed in the whole concentration range allow to precise several yielding regimes, either by Lüders band propagation for high iron concentrations $(>70 \%)$ or by homogeneous yielding for lower concentrations. In order to improve the 3D morphological characterization of these materials, 2D morphological measurements have been performed (on a large number of fields but for a narrow concentration range) thanks to an image analysis system. These measurements show that the material can be modelized by means of a boolean scheme, the parameters of which are experimentally determined. This gives access to the dependence of 3D connectivity number versus iron concentration; the good correlation between that dependence and the yielding regimes shows off the determinative influence of the space distribution on the overall plastic deformation.
\end{abstract}

\section{Introduction.}

Les efforts de modélisation en plasticité des matériaux hétérogènes ont, jusqu'à présent, essentiellement porté sur la prévision du comportement effectif global d'un hypothétique milieu homogène équivalent. Pour y parvenir, tous les modèles s'appuient sur des hypothèses, explicites ou implicites extrêmement fortes, concernant la structure. Ainsi, en traction uniaxiale, le modèle de Taylor qui repose sur une hypothèse de déformation uniforme, revient à considérer un assemblage en parallèle des constituants alors que le modèle statique, qui fait une hypothèse de contrainte uniforme, s'applique en toute rigueur à un assemblage en série des constituants ; les méthodes dites d'homogénéisation quant à elles supposent la périodicité de la structure, et les approches self-consistent n'ont de validité que dans le cas de structures parfaitement désordonnées [1].

Tant qu'on s'intéresse à une caractérisation de la réponse globale et à des régimes d'écoulement stables vis-à-vis de fluctuations des structures, ces modèles et leurs variantes semblent suffisants pour couvrir les principaux types de configurations structurales. Mais il existe des régimes d'écoulement et des propriétés ayant une très grande influence sur le comportement global et qui dépendent fortement de 
la structure géométrique du matériau comme, par exemple, les instabilités plastiques ou la fissuration en rupture ductile; à l'évidence, ces situations ne peuvent relever des approches citées ci-dessus. On se trouve en particulier dans une telle situation dans le cas de matériaux présentant une plastification par propagation de bandes de Piobert-Lüders.

Ce phénomène, couramment observé lors de la déformation en traction du fer polycristallin contenant une très faible proportion de carbone, se manifeste à la fois par l'initiation et la propagation d'un ou plusieurs fronts de plastification dans l'éprouvette (bandes de Piobert-Lüders) et par la présence d'un crochet et d'un palier sur la courbe de traction (Fig. 1) [2] ; il est clairement associé, à l'échelle microscopique, au désancrage de dislocations initialement épinglés par des défauts ponctuels. De nombreux résultats d'observation, parfois contradictoires, sont disponibles concernant la géométrie des bandes et leur cinétique de propagation ainsi que la façon dont ces caractéristiques dépendent des paramètres de l'agrégat polycristallin: taille de grains, texture. La modélisation en est cependant encore pauvre, se limitant à des aspects phénoménologiques sans qu'aucun véritable changement d'échelle n'ait été réussi sur ce mécanisme de plastification hétérogène.

Bien que l'on puisse légitimement penser que l'existence et la propagation des bandes de PiobertLüders doivent être sensibles à l'agencement géométrique des constituants de l'agrégat polycristallin, il semble qu'aucune étude expérimentale ni aucun modèle ne soit disponible.
La présente étude représente donc une première mise en évidence et caractérisation de l'influence de la morphologie et de la répartition spatiale des phases sur le comportement mécanique en plasticité des agrégats. Afin d'identifier clairement l'hétérogénéité, et aussi pour l'accuser, et dans une certaine mesure la maîtriser, des matériaux modèles ont été élaborés, constitués de fer et d'un autre matériau ne présentant pas ce phénomène. Les bandes de Piobert-Lüders sont donc utilisées comme un marqueur en tout ou rien des effets de la répartition spatiale des constituants.

Après un exposé des motifs ayant présidé au choix du matériau de l'étude et de ses principales caractéristiques, nous rappellerons quelques principes de base de l'analyse morphologique quantitative des structures biphasées désordonnées en insistant plus particulièrement sur le cas du schéma booléen. Nous présenterons ensuite les résultats des mesures morphologiques réalisés sur nos matériaux et nous en déduirons les paramètres d'un schéma booléen représentant bien leur morphologie tridimensionnelle. Nous tenterons enfin d'établir des corrélations entre comportement mécanique macroscopique et morphologie des phases constitutives.

\section{Matériau étudié.}

2.1 CHOIX DU MATÉRIAU. - L'étude reposant sur l'existence et la propagation de bandes de PiobertLüders, le choix du fer pur comme l'une des phases est imposé. L'autre phase doit être aussi peu réactive que possible avec le fer (pour ne pas former

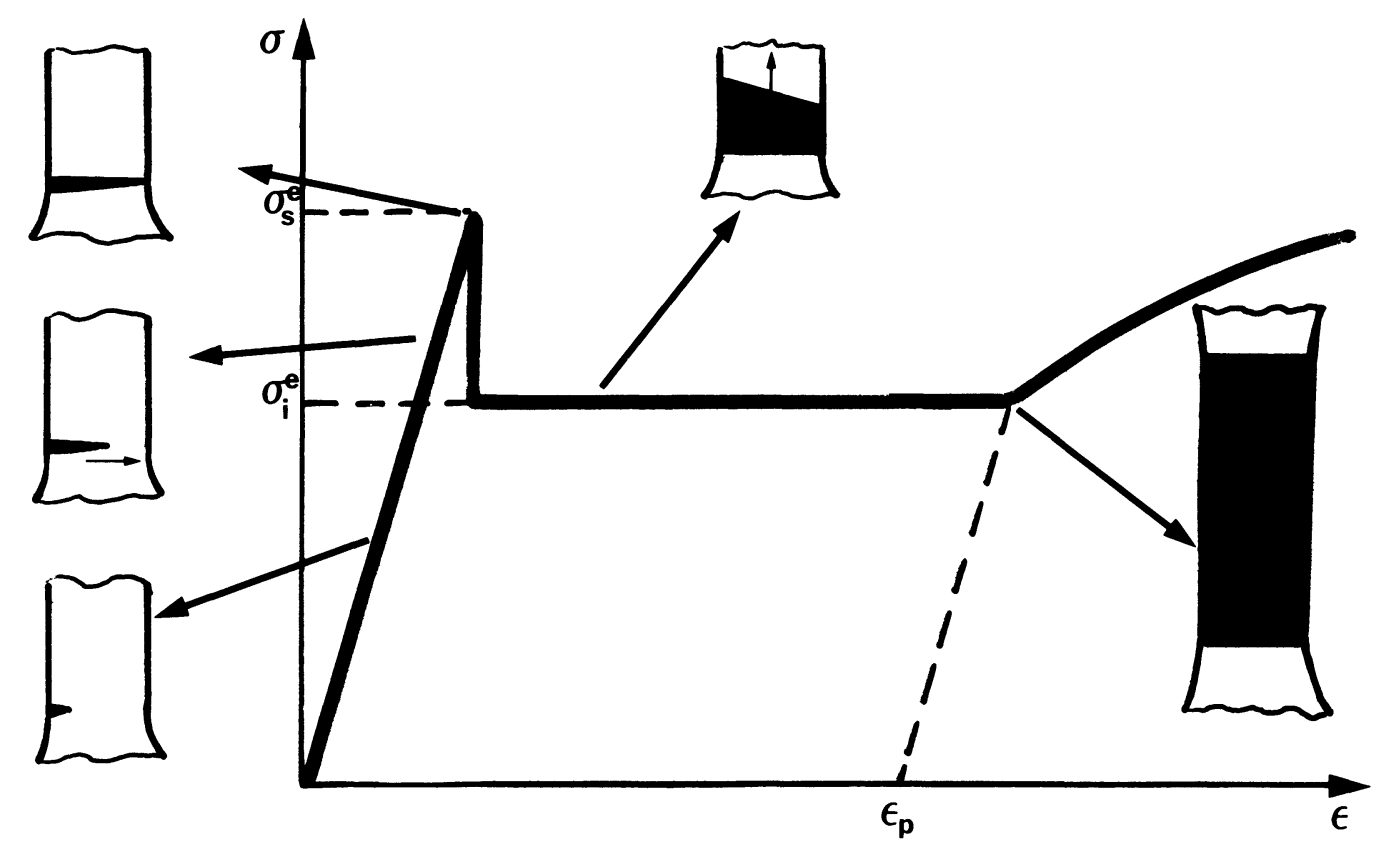

Fig. 1. - Représentation schématique de la courbe contrainte/déformation du fer et de la plastification par bande de Piobert-Lüders de l'éprouvette.

[Schematic drawing of iron stress vs. strain curve and of the yielding behaviour by Lüders band propagation.] 
d'alliage), être plastique et présenter un comportement mécanique clairement différentiable de celui $\mathrm{du}$ fer. L'argent présente des caractéristiques qui répondent bien à ces exigences.

Les matériaux de l'étude sont élaborés par compactage isostatique à chaud de mélanges de poudre de fer et d'argent. On obtient donc des matériaux composites, constitués de deux phases pures dans toute la gamme de concentration [3]. Il est possible de faire varier la morphologie des phases en faisant varier la forme et le rapport de taille des grains de poudre des deux constituants.

La présente étude s'attache à l'effet de la concentration, à morphologie des constituants constante ; les résultats évoqués ci-après ont donc été obtenus sur des matériaux élaborés à partir de poudres de fer ayant subi un tri granulométrique très serré ; seuls ont été conservés les grains compris entre 50 et $100 \mu \mathrm{m}$ (Fig. 2).

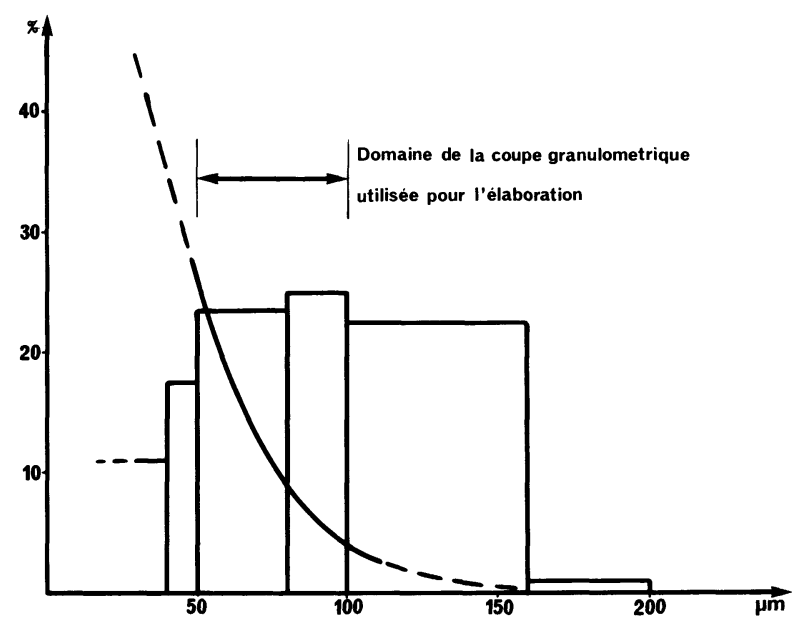

Fig. 2. - Histogramme en poids de la poudre de fer (obtenu par tamisages successifs à travers des tamis de 40 , $50,80,100,160,200 \mu \mathrm{m})$. La courbe continue représente l'allure de la répartition en taille des grains de fer comptés en nombre.

[Histogram in weight of the iron powder (it was obtained by screening the powder through screens of different mesh : 40, 50, 80,100,160, $200 \mu \mathrm{m}$ ). The continuous curve gives the distribution of iron powder grain size counted in number.]

2.2 CARACtÉRISATION DU MATÉRiau. - Des exemples de microstructures sont présentés sur la figure 3. On peut observer pour les plus fortes concentrations en fer un squelette pratiquement continu de fer constitué de sphères qui s'interpénètrent, isolant des poches d'argent (les grains de poudre d'argent se sont déformés au contact des grains de fer pendant le compactage des poudres).

Classiquement, puisqu'il s'agit de matériaux élaborés par métallurgie des poudres, la porosité a été

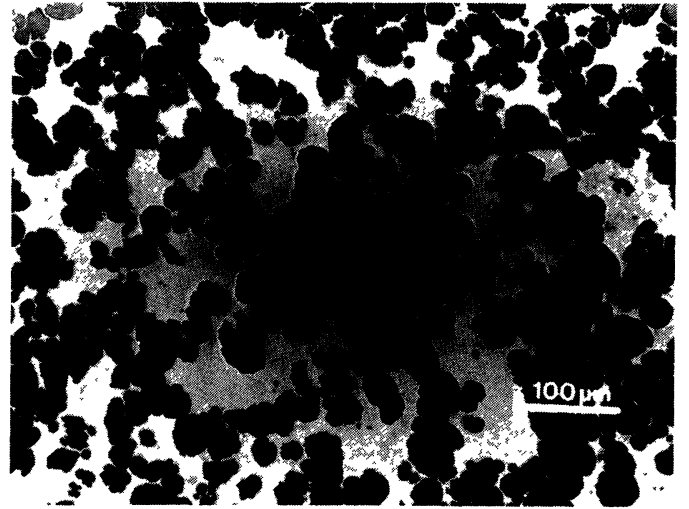

a)

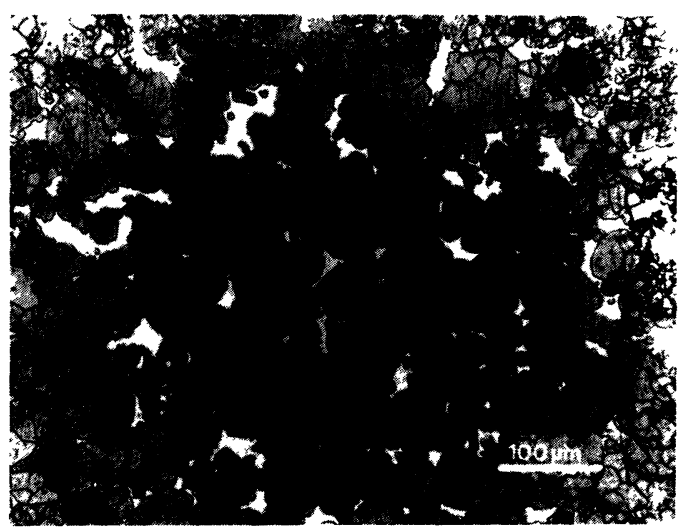

b)

Fig. 3. - Micrographies optiques de matériaux biphasés fer/argent (l'argent apparaît clair et le fer sombre) a) $50 \%$ $\mathrm{Fe}$; b) $88 \% \mathrm{Fe}$ (la microstructure du fer a été révélée par attaque chimique).

[Optical micrographs of the iron/silver two-phase materials (silver is grey and iron black). a) $50 \% \mathrm{Fe}$; b) $88 \% \mathrm{Fe}$ (the microstructure has been revealed by chemical etching).]

évaluée. Dans le fer et l'argent purs, la porosité est inférieure à la résolution des moyens classiques de mesure $(<0,1 \%)$. Dans les biphasés, la porosité semble être du même ordre; cependant il peut exister une confusion entre l'existence d'une porosité et un écart à la composition chimique théorique du matériau qui ne permet pas de totalement conclure.

La détermination de la composition en phase et la vérification de l'homogénéité sont réalisées par analyse à la microsonde électronique ; certaines éprouvettes présentant des ségrégations ( $>2 \%$ pour une aire d'analyse de $5 \times 5 \mathrm{~mm}^{2}$ ) ont été éliminées. Aucune interdiffusion aux joints d'interphase n'a pu être détectée ; compte tenu des caractéristiques de la microsonde, cela assure une zone d'interdiffusion ne dépassant pas quelques micromètres d'épaisseur [3].

Par ailleurs, l'analyse à la microsonde étant limitée à la surface, des mesures de conductivité électriques ont systématiquement été réalisées sur les éprouvettes afin de détecter d'éventuelles anomalies de composition à cœur [3]. 
Dans un faible domaine de concentration ( 9 à $18 \% \mathrm{Fe}$ ), une caractérisation morphologique quantitative a été menée sur 7 éprouvettes au moyen d'un analyseur de texture morphologique NS1500 de Nachet. Sur chaque éprouvette 20 champs jointifs au grossissement 200 ont été numérisés en microscopie optique avec une résolution de $280 \times 192$ pixels. La détermination des fonctions de corrélation (cf. paragraphe 4) a été mise en œuvre sur l'ensemble de ces champs.

2.3 COMPORTEMENT MÉCANIQUE. - On s'intéresse ici essentiellement au comportement à la plastification de nos matériaux. On peut distinguer schématiquement trois régimes de plastification (Fig. 4) :

- de $100 \%$ à $85 \%$ de fer : plastification par propagation de bandes de Lüders ;

— de $85 \%$ à $70 \%$ de fer : plastification homogène de l'argent puis plastification par bandes de Lüders ;

— moins de $70 \%$ de fer : plastification homogène de l'argent puis du fer, sans propagation de bande.

Bien que l'on se réfère ici en permanence à la concentration en phases et non à un paramètre morphologique, il est raisonnable de penser qu'un comportement mécanique aussi critique doit trouver son fondement dans l'évolution rapide de la morphologie pour de petites variations de concentration; ainsi la continuité (dans l'espace à 3 dimensions) de chacune des phases devrait permettre d'expliquer en partie le comportement mécanique macroscopique, la phase jouant un rôle de matrice (par son intercon-

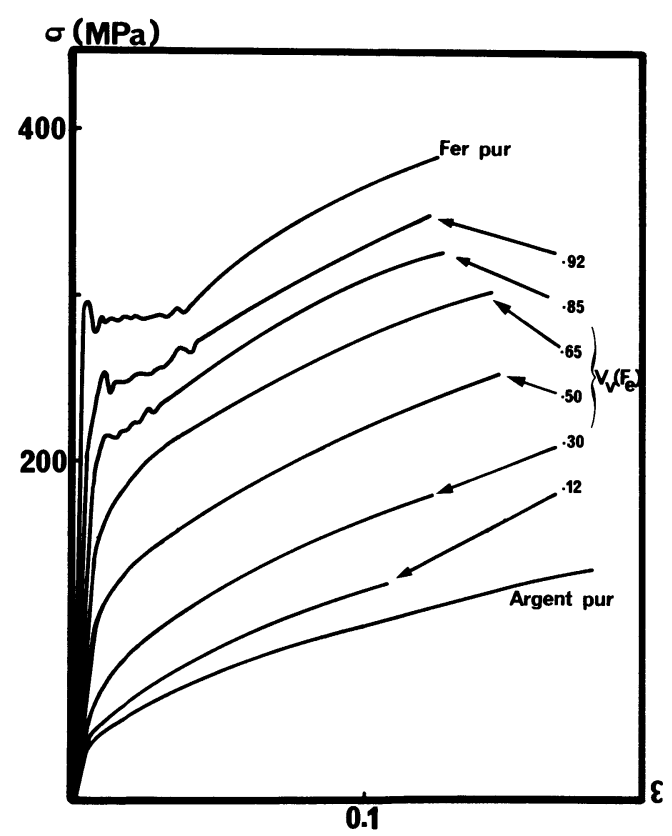

Fig. 4. - Courbes expérimentales contrainte/déformation pour toute la gamme de concentration en fer.

[Experimental stress vs. strain curves for the whole concentration range.] nexion dans l'espace) étant susceptible d'imposer ses caractéristiques propres à l'ensemble du matériau.

\section{Analyse morphologique quantitative des alliages biphasés : principes.}

Nous nous proposons dans l'analyse morphologique, de suivre une approche permettant d'appréhender la notion de continuité des phases dans l'espace, et de donner accès à ces informations par analyse d'images sur des échantillons. Par ailleurs, la modélisation de la microstructure au moyen d'un schéma booléen de sphères nous permettra de résumer toute l'information morphologique microstructurale au moyen de deux paramètres accessibles à partir de la covariance expérimentale.

\subsection{CARACTÉRISATION DE LA CONTINUITÉ DANS} L'ESPACE AU MOYEN DU NOMBRE DE CONNEXITÉ. Compte tenu de notre mode d'accès aux informations morphologiques (examens de champs au microscope optique), les contraintes classiques rencontrées en microscopie quantitative [4] limitent le choix des grandeurs morphologiques de base expérimentalement accessibles. Ainsi, dans l'espace à 3 dimensions qui nous intéresse ici, ce sont (par degrés décroissants): le volume $V$, la surface $S$, l'intégrale de la courbure moyenne $M$ et le nombre de connexité (ou intégrale de la courbure totale) $[4,5]$. Parmi ces paramètres, seul le dernier a une signification topologique, rattachée à la notion de continuité dans l'espace.

Le nombre de connexité d'une structure biphasée (ou constante d'Euler Poincaré de cette structure) est obtenu à l'aide de l'intégrale de la courbure totale (ou courbure de Gauss) sur la frontière de l'ensemble $\partial \mathrm{X}$

$$
N-G=\frac{1}{4 \pi} \int_{\partial \mathrm{X}} \frac{\partial S}{R_{1} R_{2}}
$$

Le nombre de connexité est la différence de deux termes :

- $N$ le nombre de surfaces indépendantes de l'ensemble (qui est le nombre d'objets dans le cas de particules simplement connexes sans enclaves);

- $G$ le genre qui est le nombre maximal de coupures que l'on peut effectuer sur l'objet sans le disjoindre.

A titre d'exemples, la sphère a pour nombre de connexité $1(N=1 ; G=0)$; le tore $0(N=1$; $G=1)$ et la couronne sphérique $2(N=2 ; G=0)$.

Pour une microstructure, on s'intéressera aux grandeurs spécifiques (c'est-à-dire rapportées à l'unité de volume), comme par exemple la fraction volumique $\left(V_{\mathrm{v}}\right)$, ou le nombre spécifique de connexité $\left(N_{\mathrm{v}}-G_{\mathrm{v}}\right)$. Les grandeurs spécifiques sont mesurables sur des images digitalisées par dénombre- 
ment de configurations élémentaires. On montre à l'aide de la géométrie intégrale que le nombre de connexité nécessite de connaître la structure par des coupes successives très rapprochées (cf. les algorithmes de dénombrement donnés en [4] Chap. 5), ce qui représente un travail considérable dans l'état actuel des techniques expérimentales.

N'ayant donc pas accès à une information de ce type pour cette étude, nous suivrons une autre approche, basée sur l'utilisation de modèles de structures aléatoires de type «schéma booléen », comme proposé en [9].

\subsection{UN MODÈLE DE STRUCTURE ALÉATOIRE BIPHA-} SÉE : LE SCHÉMA BOOLÉEN. - Dans cette section, nous rappelons brièvement le principe de construction de modèles d'ensembles aléatoires et l'illustrons par une introduction au schéma booléen de Matheron [6]. Cette introduction, plus intuitive que rigoureuse, est orientée vers les aspects physiques de modèles d'ensembles aléatoires. Nous invitons le lecteur intéressé par la théorie, par des développements plus complets et par des applications, à se reporter aux références $[4,7-9,11,12]$.

Un ensemble aléatoire $X$ [6-8] est caractérisé par des lois de probabilité du type :

$$
Q(\mathrm{~B}, \mathrm{x})=P\left\{\mathrm{~B}_{\mathrm{x}} \subset \mathrm{X}^{\mathrm{c}}\right\}=P\left\{\mathrm{x} \in \mathrm{X}^{\mathrm{c}} \ominus \check{\mathrm{B}}\right\}
$$

où $\check{B}$ désigne le symétrique de $\mathrm{B}$ par rapport à l'origine et $B_{x}$ est un ensemble de points (fini ou infini) translaté au point $x$.

$\mathrm{X}^{\mathrm{c}} \ominus \check{\mathrm{B}}$ est l'érodé de l'ensemble complémentaire de $\mathrm{X}$ par l'élément structurant $\mathrm{B}\left(\mathrm{X}^{\mathrm{c}} \ominus \mathrm{B}=\right.$ $\left.\left\{\mathrm{x} ; \mathrm{B}_{\mathrm{x}} \subset \mathrm{X}^{\mathrm{c}}\right\}\right)$. En pratique, pour modéliser des structures statistiquement homogènes, on utilisera des ensembles aléatoires stationnaires et ergodiques, pour lesquels $Q(\mathrm{~B}, \mathrm{x})$ est indépendant du point $\mathrm{x}$, et peut être estimé à partir d'une seule réalisation de l'ensemble aléatoire. Dans ces conditions (1) s'écrit pour $\mathrm{X} \subset \mathbb{R}^{3}$ :

$$
Q(\mathrm{~B})=V_{\mathrm{v}}\left(\mathrm{X}^{\mathrm{c}} \ominus \check{\mathrm{B}}\right) \text {. }
$$

Selon le choix de l'élément structurant (point, bipoint, segment, boule...) on accède à des informations sur la structure ayant un contenu morphologique différent (taille, répartition, anisotropie...). On considère comme identiques deux ensembles aléatoires $X_{1}$ et $X_{2}$ donnant les mêmes lois de probabilité (1) pour toutes les familles d'éléments structurants possibles. En pratique, lorsqu'on voudra identifier un modèle de structure aléatoire pour représenter une microstructure réelle comme dans cette étude on se limitera à quelques types d'éléments structurants dont on fera varier la taille.

La relation (2) conduit au mode opératoire suivant pour modéliser une microstructure par un ensemble aléatoire :
- on calcule les lois $Q(\mathrm{~B})$ en fonction des caractéristiques géométriques de $B$, de la construction du modèle et de ses paramètres. (Lorsque le calcul théorique n'est pas possible, on peut avoir recours à la mesure de $Q(\mathrm{~B})$ sur des images simulant le modèle étudié) ;

- on estime $Q(\mathrm{~B})$ sur la microstructure en procédant à des érosions et des mesures de proportion volumique par analyse d'images. L'utilisation de plusieurs familles d'éléments structurants (comme illustré plus bas) permet d'identifier un modèle (estimation de ses paramètres et tests de validité) ;

- on calcule, à l'aide du modèle, des lois $Q$ (B) (ou des caractéristiques moyennes de l'ensemble aléatoire) non accessibles directement à l'expérience (par exemple pour B tridimensionnel, lorsque le modèle a été identifié à partir d'informations planes). Cette démarche, de type "prévision » sera illustrée plus bas par l'estimation du nombre de connexité $N_{\mathrm{v}}-G_{\mathrm{v}}$.

A titre d'illustration de modèle d'ensemble aléatoire, le schéma booléen est construit comme suit (Fig. 5) :

- des germes aléatoires $x_{k}$ sont implantés dans l'espace, suivant un processus ponctuel de Poisson de densité $\theta$;

- en chaque germe est implanté un ensemble aléatoire borné $\mathrm{A}^{\prime}$ (les réalisations associées à deux germes étant indépendantes);

- l'union ensembliste $\bigcup_{\mathrm{x}_{k}} \mathrm{~A}_{\mathrm{x}_{k}}^{\prime}$ constitue l'ensem-

ble aléatoire $\mathrm{X}$.

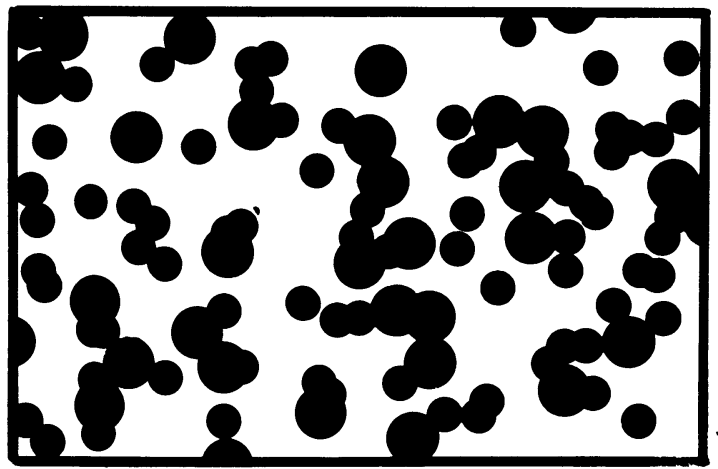

Fig. 5. - Simulation de schéma booléen par implantation « au hasard » de grains primaires $\mathbf{A}^{\prime}$ (cercles de 2 tailles différentes) dont la réunion constitue la phase $\mathbf{A}$.

[ Boolean scheme simulated by a random distribution of $\mathrm{A}^{\prime}$ primary grains (circles of two different sizes) the union of which gives the A phase.]

Ce modèle correspond à une germination $\left(\mathrm{x}_{k}\right)$ et une croissance sans interaction spatiale, puisque les grains $\mathrm{A}_{\mathrm{x}_{k}}^{\prime}$ peuvent s'interpénétrer. La comparaison des figures 3 et 5 montre la ressemblance entre les 
micrographies prises dans les alliages biphasés et une simulation.

Nous nous limiterons maintenant à quelques propriétés du schéma booléen, utiles pour la suite de l'étude.

- Les sections d'un schéma booléen de $\mathbb{R}^{n}$ par un sous-espace $\mathbb{R}^{k}$ sont un schéma booléen (de densité $\theta_{k} \neq \theta$ et de grains primaires engendrés par $\left.\mathrm{A}^{\prime} \cap \mathbb{R}^{k}\right)$. Cette propriété d'invariance stéréologique est importante pour les applications, puisqu'elle permettra de tester le caractère booléen d'une structure tridimensionnelle à partir d'informations obtenues sur des coupes planes, ou de simuler simplement des coupes de la structure à l'aide de processus ponctuels de Poisson limités à ces coupes (Fig. 5). $\mathbb{R}^{3}$ :

- La loi (2) s'écrit pour un schéma booléen de

$$
Q(\mathrm{~B})=\exp \left(-\theta \bar{V}\left(\mathrm{~A}^{\prime} \oplus \mathrm{B}\right)\right)
$$

$\bar{V}$ étant le volume moyen, pris sur l'ensemble des réalisations de $\mathrm{A}^{\prime}$. $\oplus$ étant l'opération de dilatation par B :

$$
\mathrm{X} \oplus \mathrm{B}=\left\{\mathrm{x} ; \mathrm{B}_{\mathrm{x}} \cap \mathrm{X} \neq \varnothing\right\}
$$

En pratique, on utilisera pour B plusieurs types d'éléments structurants, tels qu'un point, un couple de points ou un convexe (segment de longueur $\ell$, ou disque de rayon $r$ ) :

- pour un point

$$
q=\exp \left(-\theta \bar{V}\left(\mathrm{~A}^{\prime}\right)\right)
$$

( $q$ étant la teneur, ou fraction volumique, de $\left.\mathrm{X}^{\mathrm{c}}\right)$

- pour un couple de points séparés de la distance $h$

$$
\begin{aligned}
Q(h) & =V_{\mathrm{v}}\left(\mathrm{X}^{\mathrm{c}} \cap \mathrm{X}_{-\mathrm{h}}^{\mathrm{c}}\right) \\
& =q^{2} \exp (\theta K(h))
\end{aligned}
$$$$
\text { où } \quad K(h)=\bar{V}\left(\mathrm{~A}^{\prime} \cap \mathrm{A}_{-h}^{\prime}\right)
$$

est le covariogramme géométrique moyen de la population de grains primaires $\mathrm{A}^{\prime}$, et $Q(h)$ la covariance de l'ensemble $\mathrm{X}^{\mathrm{c}}$.

Lorsque $\ell$ est un segment de longueur $\ell$ et lorsque $\mathrm{A}^{\prime}$ est presque sûrement convexe :

$$
\bar{V}\left(\mathrm{~A}^{\prime} \oplus \ell\right)=\bar{V}\left(\mathrm{~A}^{\prime}\right)+\frac{\ell}{4} \bar{S}\left(\mathrm{~A}^{\prime}\right) .
$$

Dans les mêmes conditions ( $\mathrm{A}^{\prime}$ convexe) le nombre de connexité de $\mathrm{X}$ s'obtient par :

$$
\begin{array}{r}
N_{\mathrm{v}}(\mathrm{X})-G_{\mathrm{v}}(X)=\left[\theta-\frac{\theta \bar{M}\left(\mathrm{~A}^{\prime}\right) \theta \bar{S}\left(\mathrm{~A}^{\prime}\right)}{4 \pi}+\right. \\
\left.+\frac{\pi}{6}\left(\frac{\theta \bar{S}\left(\mathrm{~A}^{\prime}\right)}{4}\right)^{3}\right] q
\end{array}
$$

où $M$ est l'intégrale de la courbure moyenne et $S$ est l'aire dans $\mathbb{R}^{3}$.

$A$ partir des relations (3), et (8), on peut tester l'hypothèse "représentation de la structure par un schéma booléen de grains primaires convexes ", en effectuant des érosions de l'ensemble $X^{c}$ par des segments de longueur croissante $\ell(8)$. En effet dans ce cas, les courbes $Q(\ell)$ doivent avoir une forme analytique simple (fonction exponentielle linéaire en l).

A titre d'illustration la figure 6 montre les résultats obtenus pour des érosions linéaires de la phase argent, permettant d'accepter ce type de modèle, avec comme grains primaires les particules de fer.

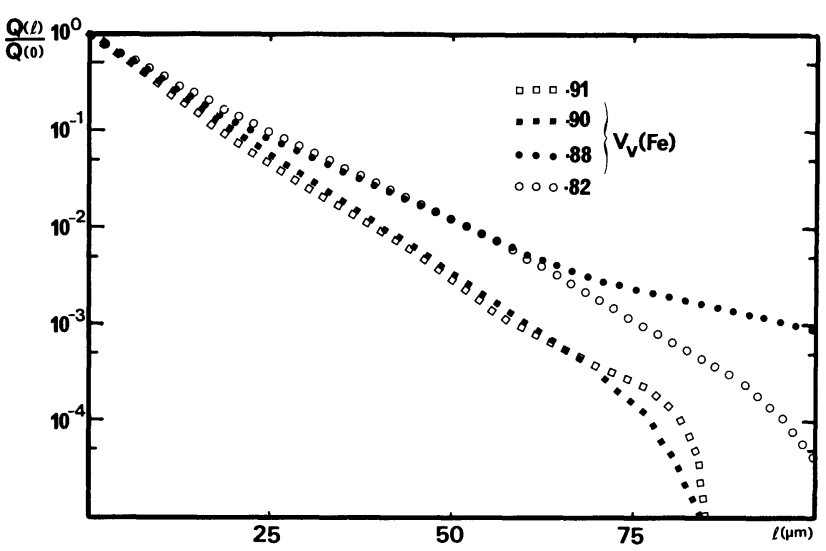

Fig. 6. - Test de l'hypothèse : « Schéma booléen à grains primaires convexes pour la phase fer ». Courbes $\log Q(1)$ pour la phase fer.

[Test of the hypothesis : «A Boolean scheme with convex primary grains can modelize iron phase ». $\log Q(1)$ curves for iron phase.]

Les mêmes relations permettent, à partir des courbes expérimentales, d'estimer $\theta \bar{S}\left(\mathrm{~A}^{\prime}\right)$, et donc d'accéder à une partie du nombre de connexité (9). L'estimation de $N_{\mathrm{v}}-G_{\mathrm{v}}$ requiert en outre la connaissance de la densité $\theta$ du schéma de points et de $\bar{M}\left(\mathrm{~A}^{\prime}\right)$. Pour y accéder, nous ferons des hypothèses supplémentaires sur la nature du grain primaire aléatoire $\mathrm{A}^{\prime}$ : comme en [9], nous considèrerons une population de sphères (dont les diamètres sont répartis suivant la loi cumulée $F(d)$ à estimer).

Dans ces conditions, on peut montrer que les paramètres du schéma booléen (c.-à-d. la densité $\theta$ et la fonction de répartition $F(d))$ sont entièrement accessibles à partir de la covariance $Q(h)(6)$.

Le modèle est donc entièrement spécifié par sa covariance, tout comme une fonction aléatoire gaussienne est connue à partir de sa fonction de corrélation d'ordre deux.

Bien qu'il soit possible théoriquement d'estimer les paramètres du modèle à partir de la seule covariance, la très mauvaise précision qu'engendre 
le calcul de dérivées secondes ou troisièmes de courbes expérimentales nous conduit à suivre la démarche adoptée en [9] où des histogrammes théoriques sont choisis a priori. A partir de ces histogrammes (le plus souvent dépendant d'un seul paramètre de taille $a$ ) on calcule le covariogramme géométrique théorique $K(h)$, que l'on compare au covariogramme expérimental réduit $K(h) / K(0)$ obtenu à partir de la relation (10) déduite de (6)

$$
\frac{K(h)}{K(0)}=2-\frac{\log Q(h)}{\log q} \text {. }
$$

Dans ces conditions, notre modèle de structure est un modèle à deux paramètres : la densité $\theta$ et le paramètre a caractérisant l'histogramme. L'ensemble des données morphologiques de la microstructure est fonction de ces deux seuls paramètres, qui réduisent considérablement l'information disponible.

Les courbes expérimentales de la figure 7 montrent les covariogrammes géométriques $K(h) / K(0)$ estimés à partir des covariances (10) pour les différents alliages étudiés. Le très bon accord entre ces données nous permet d'utiliser le même modèle de grains primaires aléatoires $\mathrm{A}^{\prime}$ (i.e. le même histogramme $F(d)$ ) pour les différentes compositions, la fraction volumique d'argent étant contrôlée par la densité $\theta$ d'implantation des grains sphériques de fer. En admettant que cette conservation du grain primaire serait vérifiée pour une gamme complète de compositions de l'alliage biphasé $\mathrm{Fe}-\mathrm{Ag}$, on pourra en déduire une courbe de variation de $N_{\mathrm{v}}-G_{\mathrm{v}}(\mathrm{Fe})$ avec $V_{\mathrm{v}}(\mathrm{Fe})($ Sect. 4).

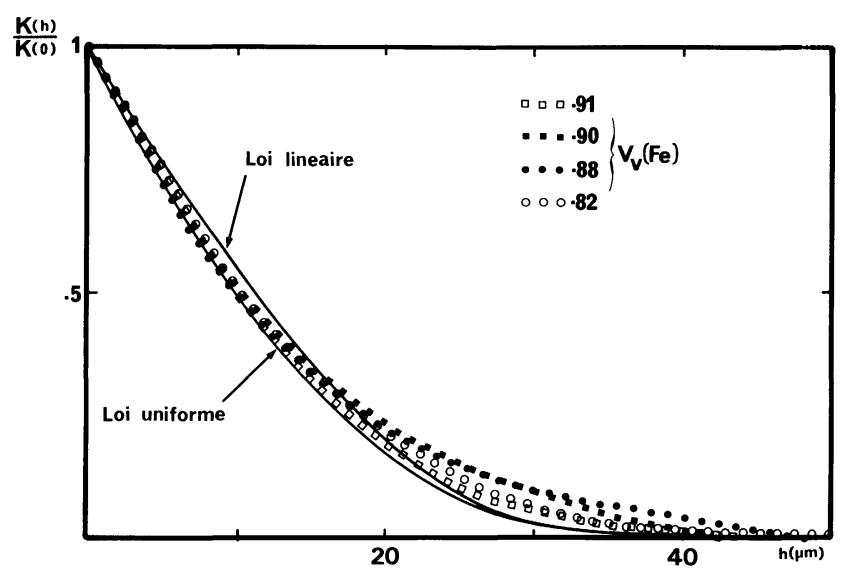

Fig. 7. - Covariogrammes géométriques expérimentaux obtenus pour 4 concentrations en fer. Les covariogrammes géométriques théoriques, de même portée $(a=40 \mu \mathrm{m})$ que les courbes expérimentales et pour deux lois de distribution en taille des grains primaires sphériques, sont également reportés.

[Experimental geometrical covariograms obtained with 4 iron concentrations. Theoretical covariograms, with the same range $(a=40 \mu \mathrm{m})$ as that of the experimental curves and for two different size distributions of the spherical primary grains, are also reported.]
La forme du covariogramme géométrique moyen d'une population de sphères se déduit du covariogramme d'une sphère et de l'histogramme des diamètres.

Les courbes théoriques obtenues pour une loi uniforme (entre 0 et $a$ ) ou pour un histogramme linéaire de même portée $a$ que les courbes expérimentales, obtenues sur les alliages étudiés, sont reportées à la figure 7 . Ces deux courbes sont très voisines, et compatibles avec nos données. Nous verrons à la section suivante que le choix de l'un ou l'autre histogramme conduit à des résultats très similaires pour l'estimation du nombre de connexité $N_{\mathrm{v}}-G_{\mathrm{v}}$ (et de ses variations avec la proportion volumique de fer), malgré la forme très différente de ces deux types d'histogramme. On peut donc s'attendre à une certaine robustesse du nombre de connexité estimé par notre méthode. Il faut également noter que la portée expérimentale utilisée à la figure $7(40 \mu \mathrm{m})$ est cohérente avec la coupe granulométrique de la poudre de fer utilisée (Fig. 2).

\section{Résultats. Relations entre morphologie et compor- tement mécanique.}

Pour les différents alliages étudiés, les paramètres du schéma booléen ont été déterminés comme suit :

- paramètre de l'histogramme de diamètre des sphères $a$ : obtenu comme portée commune des courbes $K(h) / K(0)$ des différents alliages (Fig. 7) ;

- densité $\theta$ : déduite de la mesure de teneur en $\mathrm{Ag}$, à partir de la relation (5) et du volume moyen $\bar{V}\left(\mathrm{~A}^{\prime}\right)$ correspondant à l'histogramme retenu (Tab. I).

Tableau I. - Propriétés moyennes de grains primaires sphériques du schéma Booléen ([9]).

\begin{tabular}{|l|c|c|c|}
\hline Histogramme & $\begin{array}{c}\text { Volume } \\
\text { moyen } \\
V\end{array}$ & $\begin{array}{c}\text { Surface } \\
\text { moyenne } \\
S\end{array}$ & $\begin{array}{c}\text { Intégrale } \\
\text { de la courbure } \\
\text { moyenne } M\end{array}$ \\
\hline uniforme & $\frac{\pi a^{3}}{24}$ & $\frac{\pi a^{2}}{3}$ & $\pi a$ \\
linéaire & $\frac{\pi a^{3}}{15}$ & $\frac{\pi a^{2}}{2}$ & $\frac{\pi a}{3}$ \\
uniquère & $\frac{\pi a^{3}}{6}$ & $\pi a^{2}$ & $2 \pi a$ \\
\hline
\end{tabular}

On peut ensuite déduire de la relation (9) la variation du nombre de connexité du fer avec sa proportion volumique (Fig. 8). Comme annoncé précédemment, les deux types de modèle choisis conduisent à des résultats similaires. 


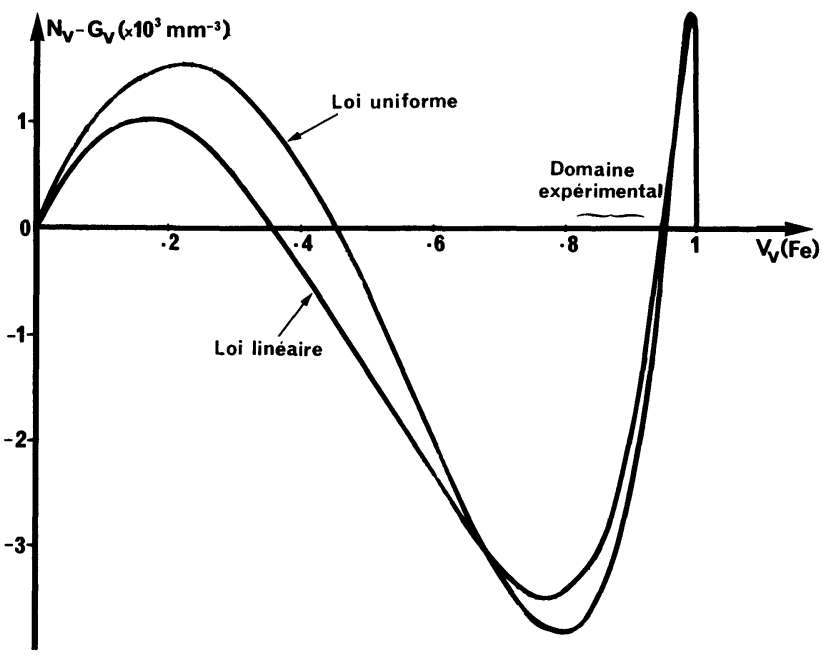

Fig. 8. - Variation du nombre de connexité tridimensionnelle du fer en fonction de la concentration en fer. Ce nombre de connexité caractérise une simulation de notre matériau par un schéma booléen; la densité et la portée de l'histogramme des diamètres des sphères ont été déterminées expérimentalement. Les deux courbes se distinguent par la loi choisie pour cet histogramme. (Les résultats d'analyse 'd'images disponibles ne permettent pas de faire un choix entre elles).

[3D connectivity number dependence versus iron concentration. This number characterizes the simulation of the material by a boolean scheme; the density and the range $a$ of the primary grain histogram has been experimentally determined. These two curves differ by the function representing that histogram (the image analysis results do not allow to discriminate between them).]

Par ailleurs, à proportion volumique identique, et pour un type de modèle fixé, une variation de la portée $a$ est compensée par une variation de la densité en sens contraire. Elle se traduit par une affinité des courbes $N_{\mathrm{v}}-G_{\mathrm{v}}$ sans changer la position des extrema et des valeurs nulles. L'interprétation qui suit, tenant compte seulement des variations de $N_{\mathrm{v}}-G_{\mathrm{v}}$ est valable pour toute microstructure du même type déduite par simple homothétie.

D'un point de vue morphologique la courbe théorique de la figure 8 , classique dans le cas du schéma booléen [9], peut s'interpréter comme suit :

- pour les faibles valeurs de la teneur en fer, le nombre de connexité est positif, les particules de fer étant assimilables à des objets simplement connexes dans une matrice d'argent ;

- après un maximum (pour $V_{\mathrm{v}}(\mathrm{Fe}) \simeq 20 \%$ ), le nombre de connexité décroît (le nombre d'interconnexions entre sphères augmente avec $\theta$, provoquant une diminution du nombre de sphères de fer isolées $\left(N_{\mathrm{v}}\right)$, et une augmentation du genre $\left(G_{\mathrm{v}}\right)$ : peu à peu la continuité du fer dans l'espace s'accentue, pendant que celle de l'argent décroît ;
- au-delà du minimum de $N_{\mathrm{v}}-G_{\mathrm{v}}$ (pour $\left.V_{\mathrm{v}} \mathrm{Fe} \simeq 0,75-0,8\right)$ le nombre de connexité augmente surtout par diminution du genre (les particules de fer envahissant tout l'espace suppriment les zones de la matrice de fer à interconnexions multiples) ;

- pour des teneurs en fer supérieures à 0,96 le matériau est constitué d'une matrice de fer comportant des inclusions d'argent.

Il est intéressant de rapprocher ces variations de propriétés topologiques du réseau de fer (et corrélativement d'argent) du comportement mécanique (Fig. 8) :

- aucune bande de Lüders n'est observée dans les alliages comportant moins de $70 \%$ de fer, correspondant (dans le cadre de notre modèle morphologique) à une microstructure constituée d'une matrice continue d'argent. Pour ce domaine de composition, le comportement de l'alliage est dicté par celui de l'argent, dont le squelette supporte les contraintes mécaniques appliquées;

- dans le domaine de teneur en fer compris entre $70 \%$ et $85 \%$, on observe deux stades : il y a d'abord plastification de l'argent, suivie de l'apparition de bandes de Lüders dans le fer. Ceci peut se comprendre par une interconnexion du fer insuffisante (en nombre de connexion et/ou en dimension de ces connexions) pour permettre la plastification de sections entières de l'éprouvette autorisant le développement de bandes de Lüders. Les sites de plastification du fer sont donc dans ce stade tout d'abord très peu nombreux et très localisés et ne font que permettre la plastification de l'argent qui s'écrouit ; le durcissement de l'argent permet alors à l'état de contrainte d'atteindre le seuil de plastification du fer dans toute une section de l'éprouvette; le fer se plastifie alors par propagation de bandes;

- pour les plus fortes teneurs $(>85 \%)$ le fer s'est constitué en un véritable squelette résistant dont les connexions sont suffisamment nombreuses et épaisses pour ne permettre aucune plastification préalable de l'argent. Quand le seuil de plasticité est atteint, ce squelette autorise la formation de bandes de Lüders.

Il semble donc que l'alliage biphasé ait le même comportement global que celui du fer pur lorsque cette phase a une interconnexion dans l'espace suffisante (c'est-à-dire au-delà du minimum de $\left.N_{\mathrm{v}}-G_{\mathrm{v}}\right)$.

Lorsque cette interconnexion n'est pas suffisante, la plastification préalable de l'argent permet d'atteindre des conditions mécaniques (écrouissage) et géométriques favorables au développement de bandes de Lüders, pourvu qu'un squelette de fer soit déjà présent (au voisinage du minimum de $N_{\mathrm{v}}-G_{\mathrm{v}}$ ).

On observe donc un bon accord qualitatif entre les seuils de teneur en fer pour l'apparition de bandes de Lüders dans les alliages biphasés $\mathrm{Fe}-\mathrm{Ag}$, et la 
courbe de variation du nombre de connexité. Celleci semble refléter la possibilité d'un phénomène de «percolation mécanique » dont la définition devrait non seulement prendre en compte l'existence de liens, mais aussi une dimension minimale de ces liens permettant la transmission d'efforts mécaniques ainsi que des propriétés mécaniques des constituants et leur évolution au cours de la déformation.

Il faut noter que les résultats classiques de calculs de percolation sur réseau discret (et non dans l'espace continu comme pour notre modèle) laisseraient prévoir un comportement du fer seul (bandes de Lüders) pour une teneur en fer variant de 0,57 à 0,805 en percolation de site ou de 0,612 à 0,881 en percolation de lien, selon le type de réseau employé [13].

\section{Conclusion.}

L'étude par analyse morphologique d'alliages biphasés fer-argent nous a permis d'utiliser un modèle d'ensemble aléatoire biphasé, le schéma booléen, pour décrire l'agencement des deux phases. A partir des deux paramètres du modèle, nous avons pu estimer le nombre spécifique de connexité, dans l'espace à trois dimensions, des microstructures, et leur variation avec la composition de l'alliage. Ces données ont permis de proposer une explication morphologique de l'apparition des bandes de Lüders au cours de la déformation en traction de ces alliages, basée sur l'interconnexion du squelette de fer.

Il conviendra de poursuivre cette première approche, en vérifiant la validité du modèle morphologique pour décrire les microstructures rencontrées sur une gamme plus étendue de compositions. Par ailleurs, comme nous l'avons déjà indiqué au paragraphe 3.2, il est possible d'effectuer des simulations de la microstructure correspondant au modèle utilisé et spécifié par les mesures réalisées par analyse d'images. Sur des simulations tridimensionnelles on pourra avoir accès numériquement à d'autres caractéristiques que la constante d'Euler-Poincaré, telle que la mesure de longueur de chemins dans chaque phase, qui paraissent hors de portée d'une caractérisation purement analytique.

Parallèlement, des efforts importants de modélisation, couplant comportement mécanique et morphologie, devront être entrepris.

\section{Remerciements.}

Les auteurs tiennent à remercier $\mathrm{V}$. Parienti et $\mathrm{P}$. Fabrègue (Centre P. M. Fourt de l'Ecole des Mines de Paris) pour leur aide dans la partie expérimentale de cette étude.

\section{Bibliographie}

[1] Zaoui A., Modelling Small Deformation of Polycrystals, chapitre 7 (Elsevier Applied Science Publishers, Amsterdam) 1986, 187-225.

[2] Jaoul B., Etude de la Plasticité et Application aux Matériaux (Dunod, Paris) 1965.

[3] Bretheau T., Caldemaison D., Feylessoufi A., Fondere J.-P., Zaoui A., J. Mater. Sci. 23 (1988) 4022-4026.

[4] Serra J., Image Analysis and Mathematical Morphology (Academic Press) 1982.

[5] HADWIGER, Vorslesungen über Inhalt, Oberfläche und Isoperimetrie (Springer, Berlin) 1957.

[6] Matheron G., Eléments pour une théorie des milieux poreux (Paris) 1967.

[7] Matheron G., Théorie des ensembles aléatoires (Ecole des Mines de Paris) 1969.
[8] Matheron G., Random Sets and Integral Geometry (J. Wiley, New York) 1975.

[9] Jeulin D., Thèse de Dr Ingénieur, Ecole des Mines de Paris (1979).

[10] HaAs A., Matheron G., Serra J., Morphologie Mathématique et Granulométries en place, Ann. Mines (1967).

[11] Jeulin D., In Special Issues of Practical Metallography, n 8, (Rierder Verlag, Stuttgart) 1978, 253263.

[12] Jeulin D., J. Microsc. Spectrosc. Electron. 12 (1987) 133-138.

[13] Clere J.-P. et al., La Percolation : modèles, simulations analogiques et numériques, Ann. Phys. Fr. 8 (1983). 